\title{
The maximum and minimum mass of protoneutron stars in the Brueckner theory
}

\author{
G. F. Burgio and H.-J. Schulze
}

\begin{abstract}
INFN Sezione di Catania and Dipartimento di Fisica, Universitá di Catania, via Santa Sofia 64, 95123 Catania, Italy e-mail: fiorella.burgio@ct.infn.it
\end{abstract}

Received 24 February 2010 / Accepted 17 April 2010

\section{ABSTRACT}

\begin{abstract}
We study the structure of protoneutron stars within the finite-temperature Brueckner-Bethe-Goldstone theoretical approach, paying particular attention to how it is joined to a low-density nuclear equation of state. We find a slight sensitivity of the minimum value of the protoneutron star mass to the low-density equation of state, whereas the maximum mass is hardly affected.
\end{abstract}

Key words. dense matter - equation of state - stars: interiors - stars: neutron

\section{Introduction}

A lot of effort is currently dedicated to dynamical simulations of supernovae explosions (Burrows \& Lattimer 1986; Pons et al. 1999; Villain et al. 2004; Liebendörfer et al. 2005; Fischer et al. 2009) and the subsequent formation and evolution of a protoneutron star (PNS). The fundamental input to these calculations is the nuclear equation of state (EOS) over a wide range of densities, apart from microscopic information regarding diffusion and cooling processes. The output are time-dependent radial profiles of the thermodynamic quantities of interest, such as temperature, entropy, particle fractions, etc.

According to current understanding, one can very roughly identify different stages of this process (Burrows \& Lattimer 1986; Pons et al. 1999; Strobel et al. 1999). (i) For about $1 \mathrm{~s}$ after supernova core bounce, the system consists of a relatively cool central region surrounded by a hot mantle, collapsing and radiating off neutrinos quickly, while still also accreting material. (ii) For the next 20 or so seconds one can identify a slowly developing state of the proper PNS, the system first deleptonizing and heating up the interior parts of the star in the process, and then beginning to cool down by further neutrino diffusion. (iii) After several tens of seconds, the final state of a cold NS has basically been formed, which continues to cool down slowly first by neutrino and later by photon emission.

In this article we do not intend to perform dynamical simulations, but focus on the consistent construction and evaluation of basic consequences of the temperature-dependent nuclear EOS during the prominent PNS stage (ii). We therefore assume strongly idealized, static profiles representing this environment. For simplicity we use a constant entropy per baryon throughout the star and investigate the sensitivity of the results to the chosen value of entropy $S / A$, as is usually done (Gondek et al. 1997; Goussard et al. 1997).

Another important aspect is the treatment of neutrino trapping. Gravitational collapse calculations of the white-dwarf core of massive stars indicate that, at the onset of trapping, the electron lepton fraction is $Y_{\mathrm{e}}=x_{\mathrm{e}}+x_{v_{\mathrm{e}}} \approx 0.4$, the precise value depending on the efficiency of electron capture reactions during the initial collapse stage. Also, because no muons are present when neutrinos become trapped, the constraint $Y_{\mu}=x_{\mu}-x_{\bar{v}_{\mu}}=0$ is imposed. We fix the $Y_{1}$ at these values in our calculations for neutrino-trapped matter. Furthermore, since low-density nuclear matter loses neutrino very fast, the concept of a neutrino sphere, inside which neutrinos are trapped, is often introduced during the PNS stage (Gondek et al. 1997; Fischer et al. 2009). Our treatment of the neutrino sphere is explained later in Sect. 4.

One of the most advanced microscopic approaches to the EOS of nuclear matter is the Brueckner theory. In recent years, it has made rapid progress in several aspects. (i) The convergence of the Brueckner-Bethe-Goldstone (BBG) expansion has been firmly established (Song et al. 1998; Baldo et al. 2000b, 2001). (ii) The addition of phenomenological three-body forces (TBF) based on the Urbana model (Carlson et al. 1983; Schiavilla et al. 1986), permitted improving the agreement to a large extent with the empirical saturation properties (Baldo et al. 1997; Zhou et al. 2004; Li et al. 2006, 2008a). (iii) The Brueckner-Hartree-Fock (BHF) approach has been extended in a fully microscopic and self-consistent way to describe nuclear matter also containing hyperons (Schulze et al. 1995, 1998). (iv) It has also been extended to the finite-temperature regime within the Bloch-De Dominicis formalism with a realistic nucleon-nucleon interaction (Baldo \& Ferreira 1999; Baldo 1999).

We used the finite-temperature BHF EOS to model PNSs in our previous papers (Nicotra et al. 2006; Burgio \& Schulze 2009). The present article represents further evolution in this approach, in particular improving two aspects of the previous work: (a) an exact calculation of the finite-temperature EOS, in contrast to the so-called "frozen correlations approximation" used before; (b) particular attention to how to join the BHF highdensity EOS to different EOSs describing the low-density domain of clustered nuclear matter.

The structure of this article is as follows. The BHF approach at finite temperature is reviewed in Sect. 2, and the resulting composition and EOS of beta-stable matter are presented in Sect. 3. The joining to different low-density EOSs is discussed in Sect. 4, and the final properties of PNSs are shown in Sect. 5. Conclusions are drawn in Sect. 6. 


\section{Brueckner theory at finite temperature}

The formalism that is closest to the BBG expansion, and that actually reduces to it in the zero-temperature limit, is the one formulated by Bloch \& De Dominicis (1958, 1959a, b). In this approach, the essential ingredient is the two-body scattering matrix $K$, which, along with the single-particle potential $U$, satisfies the self-consistent equations

$$
\begin{aligned}
& \left\langle k_{1} k_{2}|K(W)| k_{3} k_{4}\right\rangle=\left\langle k_{1} k_{2}|V| k_{3} k_{4}\right\rangle \\
& \quad+\operatorname{Re} \sum_{k_{3}^{\prime} k_{4}^{\prime}}\left\langle k_{1} k_{2}|V| k_{3}^{\prime} k_{4}^{\prime}\right\rangle \frac{\left[1-n\left(k_{3}^{\prime}\right)\right]\left[1-n\left(k_{4}^{\prime}\right)\right]}{W-E_{k_{3}^{\prime}}-E_{k_{4}^{\prime}}+\mathrm{i} \epsilon}\left\langle k_{3}^{\prime} k_{4}^{\prime}|K(W)| k_{3} k_{4}\right\rangle
\end{aligned}
$$

and

$U\left(k_{1}\right)=\sum_{k_{2}} n\left(k_{2}\right)\left\langle k_{1} k_{2}|K(W)| k_{1} k_{2}\right\rangle_{A}$,

where $k_{i}$ generally denote momentum, spin, and isospin. Here $V$ is the two-body interaction, $W=E_{k_{1}}+E_{k_{2}}$ represents the starting energy, and $E_{k}=k^{2} / 2 m+U(k)$ the single-particle energy. Equation (1) coincides with the Brueckner equation for the $K$ matrix at zero temperature if the single-particle occupation numbers $n(k)$ are taken at $T=0$. At finite temperature, $n(k)$ is a Fermi distribution. For a given density and temperature, Eqs. (1) and (2) have to be solved self-consistently along with the following equation for the auxiliary chemical potential $\tilde{\mu}$,

$\rho=\sum_{k} n(k)=\sum_{k} \frac{1}{\mathrm{e}^{\beta\left(E_{k}-\tilde{\mu}\right)}+1}$.

The grand-canonical potential density $\omega$ in the Bloch-De Dominicis framework can be written as the sum of a meanfield term and a correlation contribution (Baldo \& Ferreira 1999; Baldo 1999),

$$
\begin{aligned}
\omega= & -\sum_{k}\left[\frac{1}{\beta} \ln \left(1+\mathrm{e}^{-\beta\left(E_{k}-\tilde{\mu}\right)}\right)+n(k) U(k)\right] \\
& +\frac{1}{2} \int \frac{\mathrm{d} W}{2 \pi} \mathrm{e}^{\beta(2 \tilde{\mu}-W)} \operatorname{Tr}_{2}\left(\arctan \left[\mathcal{K}(W) \pi \delta\left(H_{0}-W\right)\right]\right),
\end{aligned}
$$

where the trace, $\operatorname{Tr}_{2}$, is taken in the space of antisymmetrized two-body states, and the two-body scattering matrix $\mathcal{K}$ is defined by

$$
\left\langle k_{1} k_{2}|\mathcal{K}(W)| k_{3} k_{4}\right\rangle=\left\langle k_{1} k_{2}|K(W)| k_{3} k_{4}\right\rangle \prod_{i=1,4} \sqrt{1-n_{i}(k)} .
$$

If one only considers the first term in the expansion of the arctan function (Baldo \& Ferreira 1999, see Appendix A), then the correlation term reduces to

$\omega_{\mathrm{c}}=\frac{1}{2} \sum_{k} n(k) U(k)$,

which defines the grand-canonical potential in total analogy with the BBG binding potential, just using Fermi functions instead of the usual step functions at zero temperature. In this way one neglects a series of terms proportional to $n(k)[1-n(k)]$ (or powers of it), which turn out to be negligible in the temperature and density ranges relevant for neutron and protoneutron stars.

A further simplification can be achieved by disregarding the effects of finite temperature on the single-particle potential $U$, and using the $T=0$ results in order to speed up the calculations (frozen correlations approximation). This was the procedure followed in our previous publications (Nicotra et al. 2006; Burgio $\&$ Schulze 2009). On the contrary, all calculations shown in this paper are obtained without introducing any approximation on the single-particle potential $U$, and using the exact expression for the grand-canonical potential, Eq. (4).

Furthermore, in the previous work the calculations were performed using the $G$-matrix instead of the $K$-matrix, [i.e., taking the real part in Eq. (2) instead of Eq. (1)], which produces a more repulsive EOS at high density. Strictly speaking, the Bloch-De Dominicis formalism has been derived for the $K$-matrix; on the other hand, the use of the $G$-matrix ensures the compatibility with the usually performed continuous-choice BHF calculations at zero temperature.

In this framework, the free energy density is then

$f=\omega+\rho \tilde{\mu}$,

and all other thermodynamic quantities of interest can be computed from it; namely, the "true" chemical potential $\mu$, pressure $p$, entropy density $s$, and internal energy density $\epsilon$ read as

$\mu=\frac{\partial f}{\partial \rho}$

$p=\rho^{2} \frac{\partial(f / \rho)}{\partial \rho}=\mu \rho-f$,

$s=-\frac{\partial f}{\partial T}$,

$\epsilon=f+T s$.

We stress that this procedure permits to fulfill the HugenholtzVan Hove theorem in the calculation of thermodynamical quantities in the Brueckner theory. For an extensive discussion of this topic, the reader is referred to Baldo (1999), and references therein.

It is well known that at zero temperature the non-relativistic microscopic approaches do not correctly reproduce the nuclear matter saturation point $\rho_{0} \approx 0.17 \mathrm{fm}^{-3}, E / A \approx-16 \mathrm{MeV}$. One common way of correcting this deficiency is to introduce threebody forces among nucleons. Since a complete microscopic theory of TBF is not available yet, we have adopted the phenomenological Urbana model (Carlson et al. 1983; Schiavilla et al. 1986), which consists of an attractive term due to two-pion exchange with excitation of an intermediate $\Delta$ resonance and a repulsive phenomenological central term. In the BBG approach, the TBF is reduced to a density-dependent two-body force by averaging over the position of the third particle, assuming that the probability of having two particles at a given distance is reduced according to the two-body correlation function. The corresponding EOS reproduces the nuclear matter saturation point correctly (Baldo et al. 1997; Zhou et al. 2004; Li et al. 2008a) and fulfills several requirements from the nuclear phenomenology. In all calculations presented in this paper we used the Argonne $V_{18}$ nucleon-nucleon potential (Wiringa et al. 1995) along with the phenomenological Urbana TBF.

In Fig. 1 we display the free energy vs. the nucleon density, obtained following the procedure discussed above, both for symmetric and pure neutron matter, and for several values of the temperature between 0 and $50 \mathrm{MeV}$. The dots represent the numerical results, and the curves are empirical fits, which can be easily implemented in numerical simulations. The thin dashed curves represent the calculations obtained with the $G$-matrix and the frozen correlations approximation for the single-particle potential $U$, as adopted in our previous papers (Nicotra et al. 2006; 


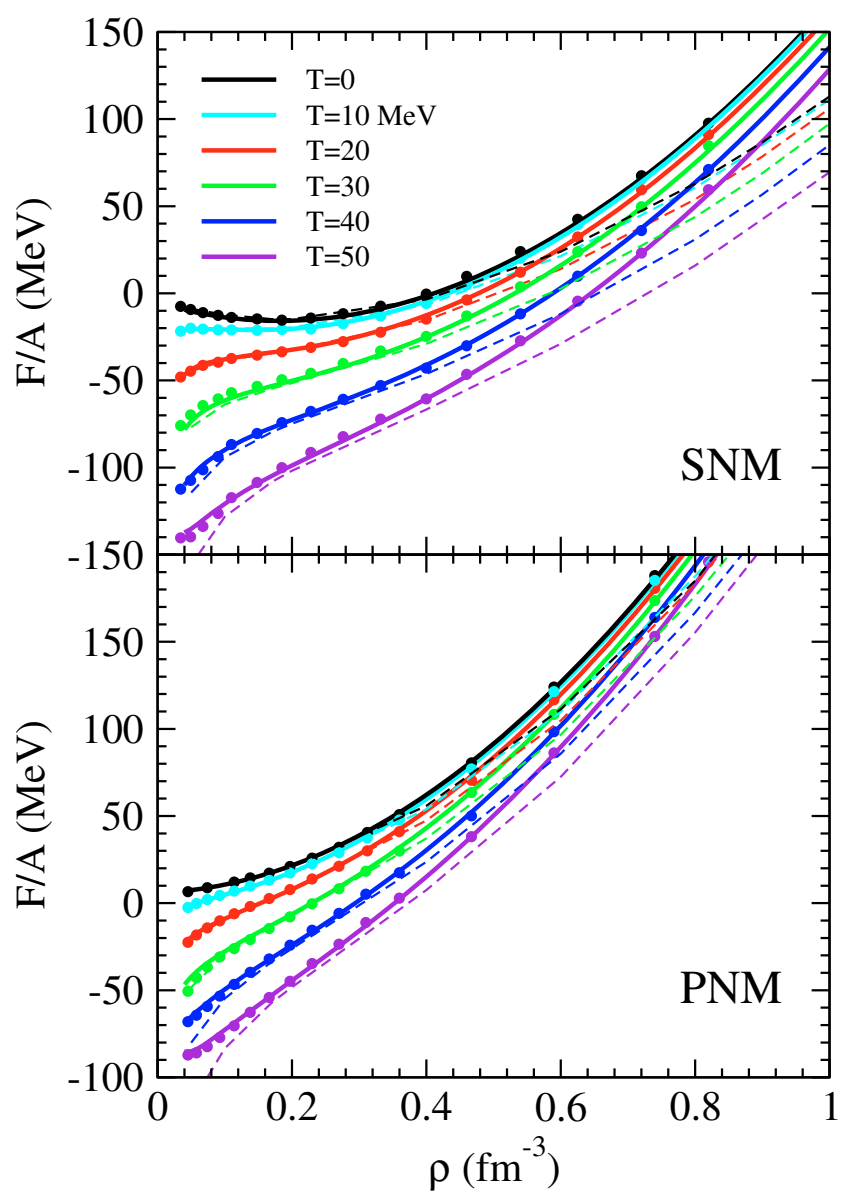

Fig. 1. Free energy per nucleon for symmetric (upper panel) and pure neutron (lower panel) matter (solid lines). The temperatures vary from 0 to $50 \mathrm{MeV}$ in steps of $10 \mathrm{MeV}$. See text for details.

Burgio \& Schulze 2009). The discrepancy at high density is mainly due to the use of the $G$-matrix instead of the $K$-matrix, whereas the differences observed at low density and high temperature are consequences of the frozen correlations approximation.

We find that the following functional forms provide excellent parametrizations of the new numerical results in the required ranges of density $\left(0.03 \mathrm{fm}^{-3} \lesssim \rho \lesssim 1 \mathrm{fm}^{-3}\right)$ and temperature ( $0 \mathrm{MeV} \leq T \leq 50 \mathrm{MeV})$ for symmetric (SNM) and pure neutron matter (PNM):

$$
\begin{aligned}
\frac{F}{A}(T, \rho)= & -\left(137+157 t^{2}\right) \rho+308 \rho^{1.82}+207 t^{2} \ln (\rho) \\
& +\left(-47.5 t^{2}+71 t^{2.41}\right) / \rho-5 \quad(\mathrm{SNM}), \\
\frac{F}{A}(T, \rho)= & \left(11-122 t^{2}\right) \rho+309 \rho^{1.95}+173 t^{2} \ln (\rho) \\
& +\left(-48 t^{2}+71 t^{2.35}\right) / \rho+6 \quad(\mathrm{PNM}),
\end{aligned}
$$

where $t=T /(100 \mathrm{MeV})$ and $F$ and $\rho$ are given in $\mathrm{MeV}$ and $\mathrm{fm}^{-3}$, respectively.

We notice that the free energy of symmetric matter shows a typical Van der Waals behavior (with $T_{\mathrm{C}} \approx 19 \mathrm{MeV}, \rho_{\mathrm{c}} \approx$ $0.06 \mathrm{fm}^{-3}$ ) and is a monotonically decreasing function of the temperature. At $T=0$ the free energy coincides with the total energy and the corresponding curve is just the usual nuclear matter saturation curve.

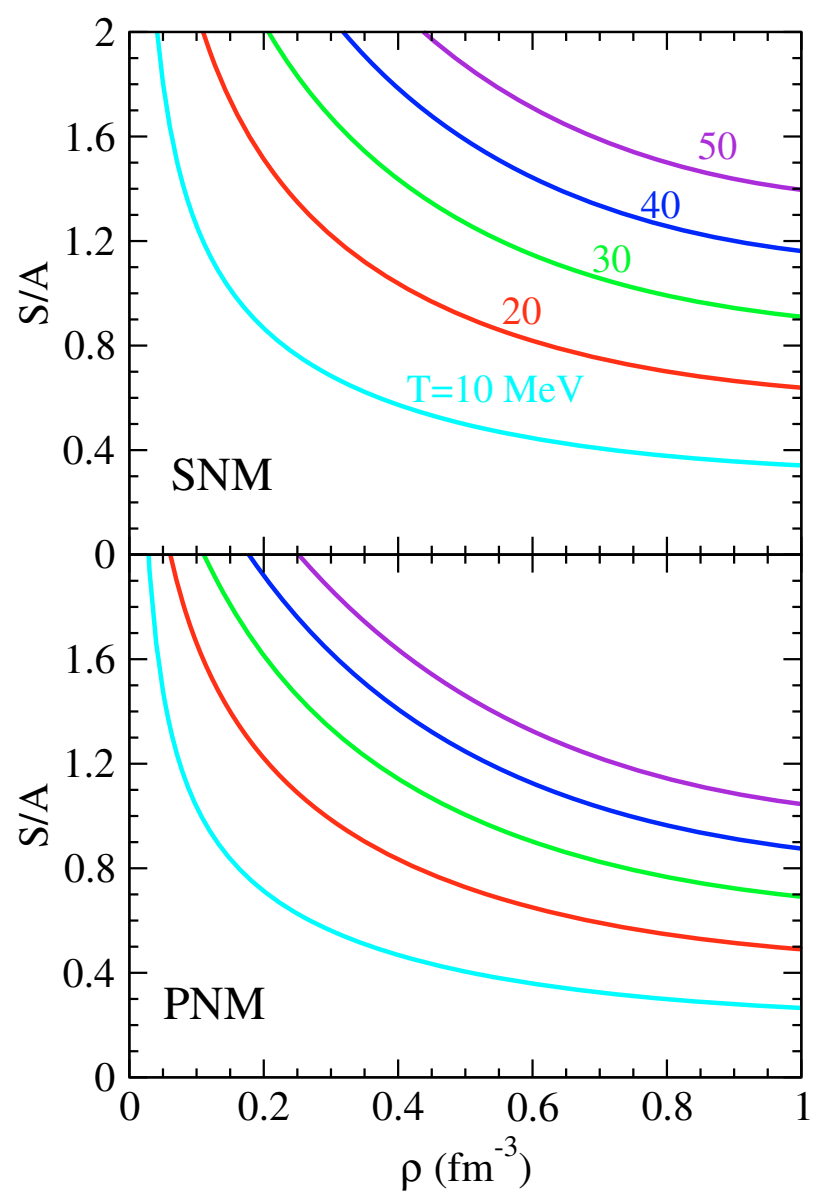

Fig. 2. The entropy per particle for symmetric (upper panel) and pure neutron (lower panel) matter at different temperatures.

From the free energy we can calculate the entropy (in units of the Boltzmann constant) from the thermodynamical relationship

$\frac{S}{A}(\rho)=-\left(\frac{\partial F / A}{\partial T}\right)_{\rho}$

and this is displayed in Fig. 2 for both SNM and PNM.

It turns out that the dependence of the free energy on the proton fraction can be approximated very well by a quadratic dependence, as at zero temperature (Bombaci \& Lombardo 1991; Baldo et al. 1998, 2000a):

$\frac{F}{A}(T, \rho, x) \approx \frac{F}{A}(T, \rho, x=0.5)+(1-2 x)^{2} F_{\mathrm{sym}}(T, \rho)$,

where the symmetry energy $F_{\text {sym }}$ can be expressed in terms of the difference of the energy per particle between pure neutron $(x=0)$ and symmetric $(x=0.5)$ matter:

$$
\begin{aligned}
F_{\mathrm{sym}}(T, \rho) & =-\frac{1}{4} \frac{\partial(F / A)}{\partial x}(T, \rho, 0) \\
& \approx \frac{F}{A}(T, \rho, 0)-\frac{F}{A}(T, \rho, 0.5) .
\end{aligned}
$$

Therefore, in order to treat the beta-equilibrated case, it is only necessary to use the parametrizations Eqs. $(12,13)$ of the free energy for SNM and PNM.

For completeness, we display in Fig. 3 the behavior of the free symmetry energy for several values of the temperature. Full dots represent the numerical values, and the solid curves are the results of the polynomial fits. Even at finite temperature, the 


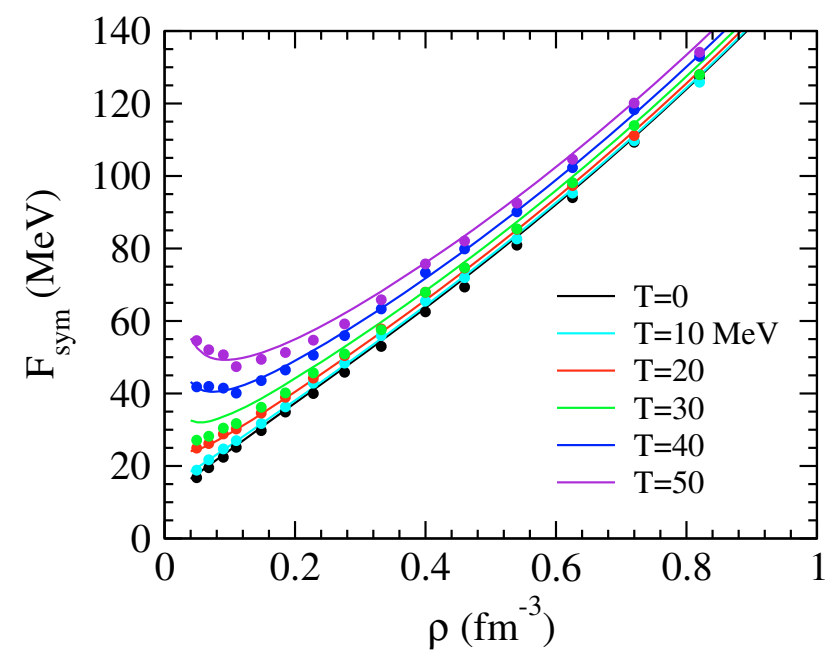

Fig. 3. The free symmetry energy as a function of the nucleon density at different temperatures.

symmetry energy increases nearly linearly with density, as is generally the case in the BHF approach (Li et al. 2006, 2008a). However, at low density there are significant deviations due to finite temperature, which might have experimental implications. The symmetry energy plays a significant role not only in the cooling of protoneutron stars, but also in the dynamics of heavy ion collisions induced by radioactive beams and the structure of exotic nuclei. For a review, see Li et al. (2008b).

\section{Composition and EOS of hot stellar matter}

In neutrino-trapped $\beta$-stable nuclear matter, the chemical potential of any particle $i=\mathrm{n}, \mathrm{p}, 1$ is uniquely determined by the conserved quantities baryon number $B_{i}$, electric charge $Q_{i}$, and weak charges (lepton numbers) $L_{i}^{(\mathrm{e})}, L_{i}^{(\mu)}$ :

$\mu_{i}=B_{i} \mu_{n}-Q_{i}\left(\mu_{\mathrm{n}}-\mu_{\mathrm{p}}\right)+L_{i}^{(\mathrm{e})} \mu_{v_{\mathrm{e}}}+L_{i}^{(\mu)} \mu_{v_{\mu}}$.

For stellar matter containing nucleons and leptons as relevant degrees of freedom, the chemical equilibrium conditions read explicitly as

$\mu_{\mathrm{n}}-\mu_{\mathrm{p}}=\mu_{\mathrm{e}}-\mu_{v_{\mathrm{e}}}=\mu_{\mu}+\mu_{\bar{v}_{\mu}}$.

At given baryon density $\rho$, these equations have to be solved with the charge neutrality condition

$\sum_{i} Q_{i} x_{i}=0$

and those expressing conservation of lepton numbers

$Y_{1}=x_{1}-x_{\overline{1}}+x_{\nu_{1}}-x_{\bar{\nu}_{1}}, \quad 1=\mathrm{e}, \mu$.

As discussed in Sect. 1, we fix the lepton fractions to $Y_{\mathrm{e}}=0.4$ and $Y_{\mu}=0$ for neutrino-trapped matter. When the neutrinos have left the system, their partial densities and chemical potentials vanish and the above equations simplify accordingly.

The nucleon chemical potentials are obtained from the free energy density $f$, Eq. (7),

$\mu_{i}\left(\left\{\rho_{j}\right\}\right)=\left.\frac{\partial f}{\partial \rho_{i}}\right|_{\rho_{j \neq i}}, i=\mathrm{n}, \mathrm{p}$,

and the chemical potentials of the non-interacting leptons are obtained by solving numerically the free Fermi gas model at finite temperature. Once the hadronic and leptonic chemical potentials are known, one can proceed to calculate the composition of the $\beta$-stable stellar matter, and then the total pressure $p$ through the usual thermodynamical relation

$p=\rho^{2} \frac{\partial(f / \rho)}{\partial \rho}=\sum_{i} \mu_{i} \rho_{i}-f$.

Following this procedure, we first discuss the populations of beta-equilibrated stellar matter. In Fig. 4 we display the relative particle fractions as a function of the baryon density for cold (left panels) and hot beta-stable matter characterized by entropy values $S / A=1$ (central panels) and $S / A=2$ (right panels). The upper panels show the particle fractions when stellar matter contains only neutrons, protons, electrons, and muons, whereas the lower panels show the particle fractions in neutrino-trapped matter. We notice that the electron fraction is greater in neutrinotrapped matter than in the neutrino-free case; therefore, the nuclear matter is more symmetric, and the resulting EOS will be softer. The appearance of muons in trapped matter is shifted to higher density values, because their onset is determined by the difference between the neutron and proton chemical potentials, Eq. (19).

We observe that thermal effects influence the populations mainly in the low-density region. In fact, the presence of tails in the Fermi distribution makes it possible to create (anti)particles at any density and thus typical production thresholds, like for muon creation, disappear at finite entropy. At high density, thermal effects are less important and do not change the composition appreciably when increasing entropy. As already found in (Nicotra et al. 2006) in the isothermal case and in (Burgio \& Schulze 2009), the proton fraction increases slightly at high density with increasing entropy, thus causing a softening of the EOS. This is at variance with the results shown in (Prakash et al. 1997), and is presumably caused by the different temperature dependence of the potential part of the EOS.

In Fig. 5, we display the pressure for beta-stable asymmetric matter with and without neutrinos, as a function of the energy density at entropy $S / A=2$ (red curves), in comparison with cold beta-stable matter (black curves). As discussed above, we notice that both thermal effects and neutrino trapping produce a softer EOS than in the cold case.

\section{Low-density EOS}

The Brueckner approach provides a realistic modeling of nuclear matter only at densities above about half normal nuclear matter density. Below this threshold, clusterization sets in and the system becomes inhomogeneous. Another theoretical approach therefore has to be used in this "low-density" regime, and we employ two widely used liquid-drop-type models at finite temperature, namely the one of Lattimer \& Swesty (1997) and the one of Shen et al. (1998a, b). In the first case we in fact compare a model with a (too) large compressibility, $K=370 \mathrm{MeV}$, here denoted by "LS" and a second one with a lower compressibility, $K=263 \mathrm{MeV}$, denoted by "SKa". The Shen EOS is characterized by a compressibility $K=281 \mathrm{MeV}$ and a symmetry energy at saturation of $E_{\text {sym }}=36.9 \mathrm{MeV}$, while the BHF EOS has $K=210 \mathrm{MeV}$ and $E_{\text {sym }}=34.7 \mathrm{MeV}$.

Of course, since no phase transition is involved, but only two different theoretical descriptions of the same state of matter, the joining of the two EOSs requires the thermodynamical observables, i.e., free energy, internal energy, pressure, and chemical potentials, to be continuous functions of the baryon density. Of 


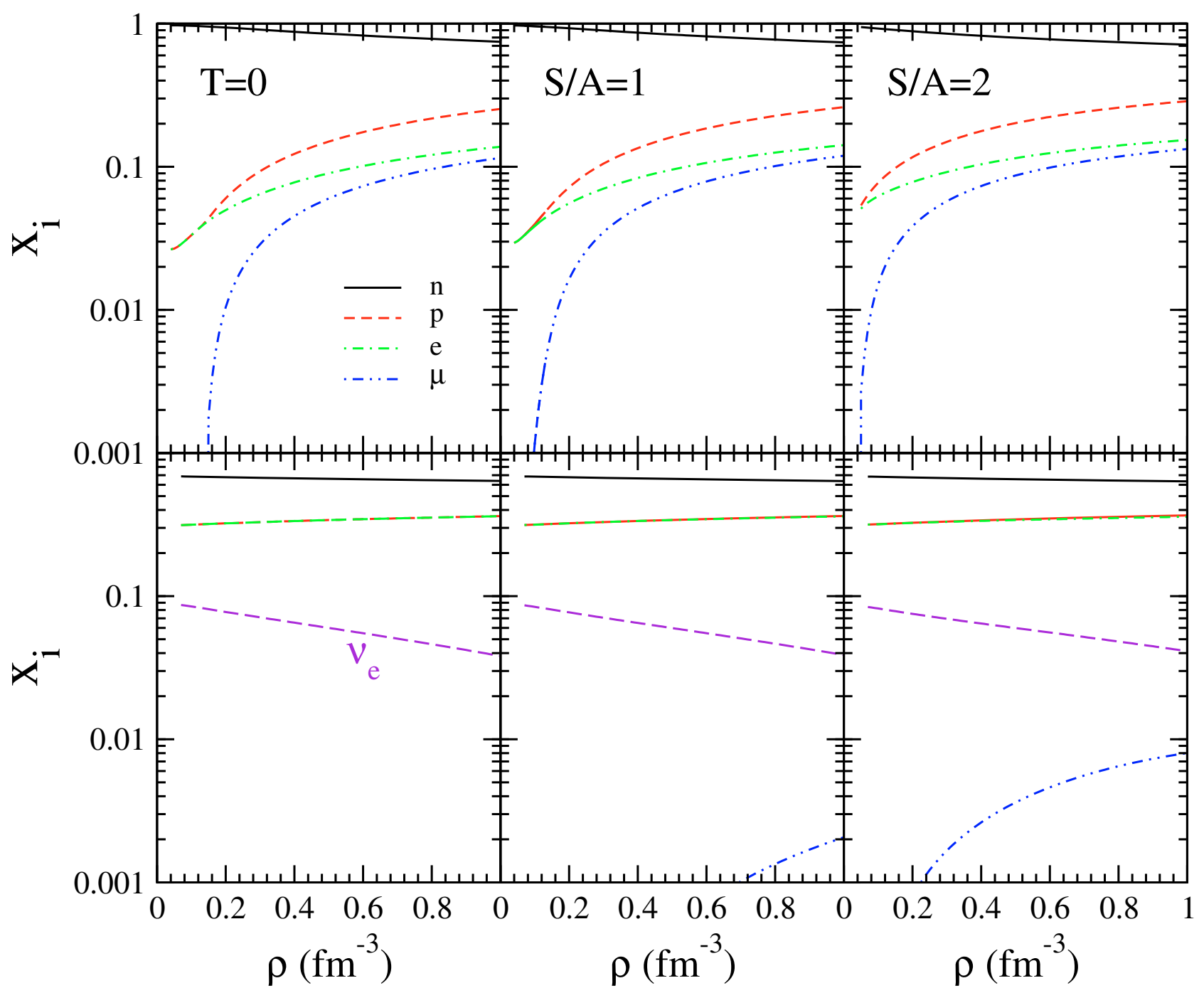

Fig. 4. Relative populations as functions of baryon density in neutrino-free (upper panels), and neutrino-trapped (lower panels) beta-equilibrated matter at entropies $S / A=0,1,2$.

particular interest are therefore (i) the consistent joining of the low- and high-density EOS, and (ii) eventual differences caused by the use of different low-density models. These aspects are studied in detail in the following.

Another important feature of the low-density domain is the treatment of neutrino trapping. Physically, neutrinos escape rapidly from the low-density matter during the PNS evolution, and so the trapping condition $Y_{\mathrm{e}}=0.4$ does not apply anymore. This effect can be roughly modeled by the concept of a neutrino sphere inside which the neutrinos are trapped. Typical modeldependent values for the location of the neutrino sphere found in the literature are $2 \times 10^{-3} \mathrm{fm}^{-3}$ (Gondek et al. 1997), $6 \times$ $10^{-4} \mathrm{fm}^{-3}$ (Strobel et al. 1999), and $2 \times 10^{-5} \mathrm{fm}^{-3}$ (Fischer et al. 2009). Given these variations, we choose the following "natural cutoff" procedure: by imposing the condition $Y_{\mathrm{e}}=0.4$ at any density, at a certain threshold density $\rho_{v} \approx 10^{-5}-10^{-6} \mathrm{fm}^{-3}$, the electron fraction $x_{\mathrm{e}}$ becomes 0.4 , and the neutrinos disappear naturally. For lower densities we consider the matter untrapped. This is illustrated in Fig. 6, showing the electron and neutrino fractions following this recipe with the different EOSs. This simple procedure avoids making assumptions about the neutrino sphere, but clearly a satisfactory treatment of neutrino trapping would require coupled dynamical calculations of PNS evolution and the temperature-dependentEOS, which might be possible in the future.

In Fig. 7, we display the joint low-density LS, SKa, Shen + BHF EOSs over a wide range of densities. The black lines represent the calculations performed at $T=0$, whereas the red (blue) lines are the ones for fixed entropy $S / A=1(S / A=2)$ in neutrino-trapped matter. The transition densities between lowdensity inhomogeneous regime and homogeneous BHF regime in the different configurations are displayed in Fig. 7 as vertical bars, and summarized in Table 1. One observes in general a reduction of the transition density with decreasing compressibility of the low-density EOS and with increasing temperature. In all configurations, a consistent joining of the two segments of the EOS is possible, fulfilling the above-mentioned continuity requirements. In practice we performed a Maxwell construction by equating pressure and chemical potentials of the low- and high-density sectors, and verified that the other thermodynamic variables do not exhibit significant discontinuities at the transition point.

As a further general prominent feature, we notice a strong stiffening of the EOS in neutrino-trapped matter as compared to the cold untrapped case in the domain $\rho \approx 10^{-4}, \ldots, 10^{-1} \mathrm{fm}^{-3}$ (black vs. colored curves in Fig. 7), which comes from the 


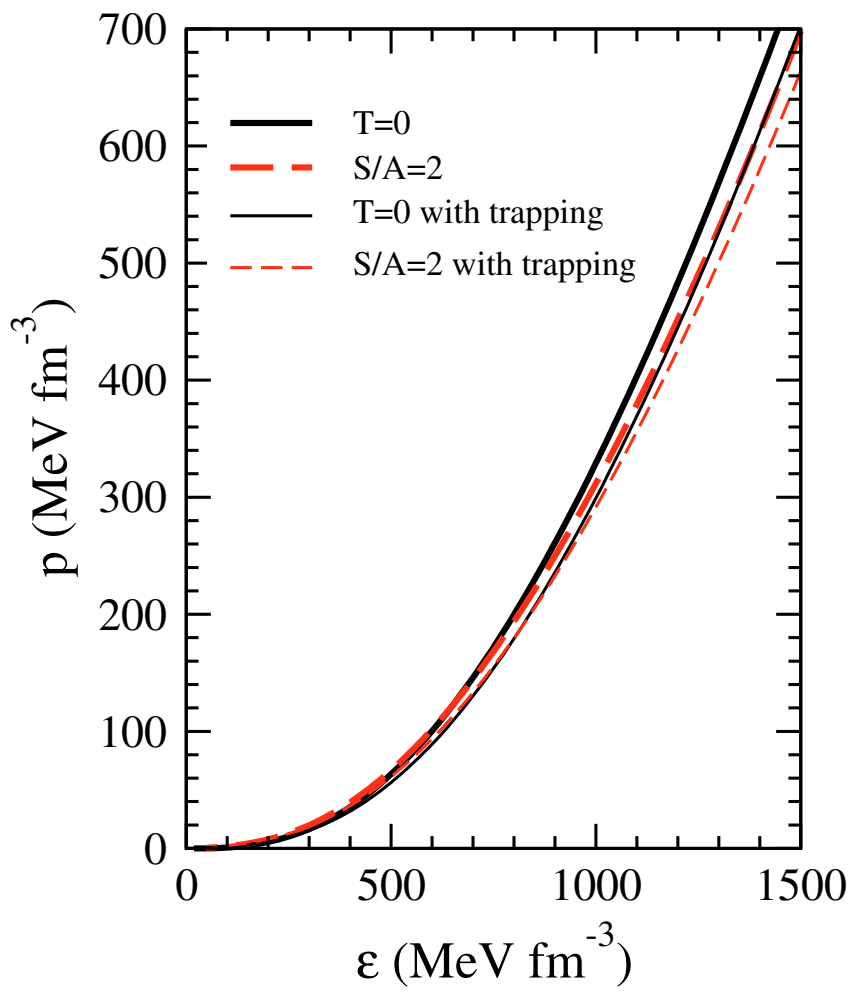

Fig. 5. Pressure as a function of energy density for beta-equilibrated cold and hot matter with and without neutrino trapping.

Table 1. Transition densities (in $\mathrm{fm}^{-3}$ ) between low-density (LS, SKa, Shen) and BHF EOS.

\begin{tabular}{llll}
\hline \hline & Untrapped, $T=0$ & Trapped, $S / A=1$ & Trapped, $S / A=2$ \\
\hline LS & 0.160 & 0.145 & 0.130 \\
SKa & 0.110 & 0.090 & 0.086 \\
Shen & 0.120 & 0.110 & 0.094 \\
\hline
\end{tabular}

greater lepton Fermi pressure in trapped matter (larger electron fraction and additional neutrino contribution, cf., Fig. 6).

\section{5. (Proto)Neutron star configurations}

The stable configurations of a (P)NS can be obtained from the well-known hydrostatic equilibrium equations of Tolman, Oppenheimer, and Volkov (Shapiro \& Teukolsky 1983) for pressure $p(r)$ and enclosed mass $m(r)$,

$$
\begin{aligned}
\frac{\mathrm{d} p}{\mathrm{~d} r} & =-\frac{G m \epsilon}{r^{2}} \frac{(1+p / \epsilon)\left(1+4 \pi r^{3} p / m\right)}{1-2 G m / r} \\
\frac{\mathrm{d} m}{\mathrm{~d} r} & =4 \pi r^{2} \epsilon
\end{aligned}
$$

once the $\operatorname{EOS} p(\epsilon)$ is specified, with $\epsilon=\epsilon_{\mathrm{N}}+\epsilon_{\mathrm{L}}$ the total internal energy density ( $G$ is the gravitational constant). For a given central value of the energy density, the numerical integration of Eqs. (24) and (25) provides the mass-radius relation.

Our results, using the different EOSs introduced in the previous section, are displayed in Fig. 8, which shows the gravitational mass (in units of the solar mass $M_{\odot}=1.98 \times 10^{33} \mathrm{~g}$ ) as a function of the radius (left panels), and the central density (right panels) (normalized with respect to the saturation value $\epsilon_{0}=152 \mathrm{MeV} \mathrm{fm}^{-3}$ ). The black curves represent the calculations performed at $T=0$, whereas the thick (thin) colored lines

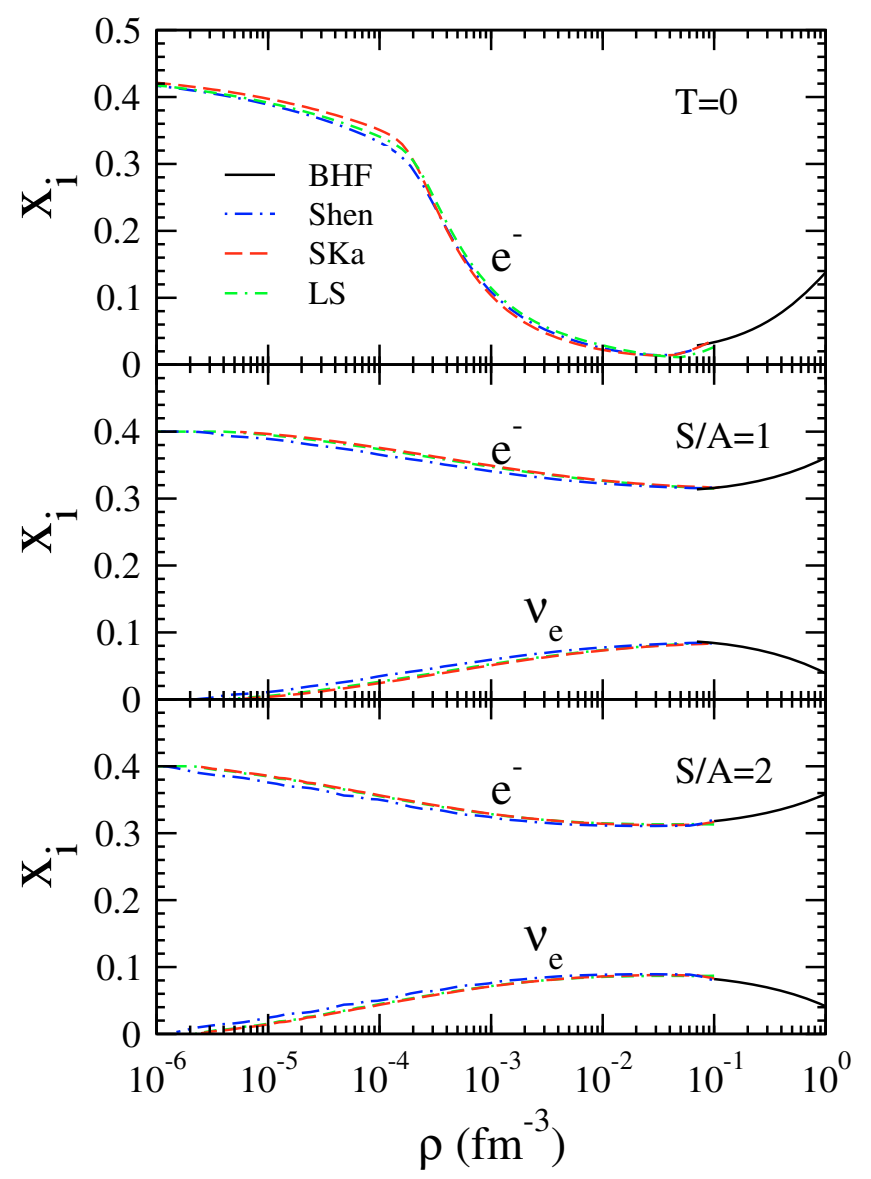

Fig. 6. The lepton fractions in beta-stable matter as function of baryon density for the different EOSs. denote stable configurations in neutrino-free (neutrino-trapped) matter at constant entropy $S / A=1$ (dashed lines) and $S / A=2$ (dot-dashed lines).

We notice that the value of the maximum mass decreases slightly in neutrino-trapped matter, because of the softening of the EOS discussed before. The value of the maximum mass turns out to be practically independent of the entropy, whereas the value of the radius for a fixed gravitational mass depends strongly on the entropy and on the presence of neutrinos. The dots mark the values of the minimum mass and the corresponding radius a PNS may have, with typical values in the range $0.6-0.77 M_{\odot}$ and about $40-50 \mathrm{~km}$, according to the chosen lowdensity EOS. The proper values are summarized in Table 2. One notes that the minimum mass configurations only probe the lowdensity part of the EOS. As expected, the minimum mass increases with decreasing compressibility of the EOS, and with increasing entropy of the matter.

Our values of the minimum mass are very similar to the ones found in Gondek et al. (1997), where the LS EOS was used over the full density range of the PNS. On the other hand, our values turn out to be lower than those calculated in Strobel et al. (1999), the reason being that our minimum mass is calculated during stage (ii) in the PNS evolution, when neutrinos are still trapped, and the core and the mantle are characterized by the same entropy values as described in the introduction. 
G. F. Burgio and H.-J. Schulze: The maximum and minimum mass

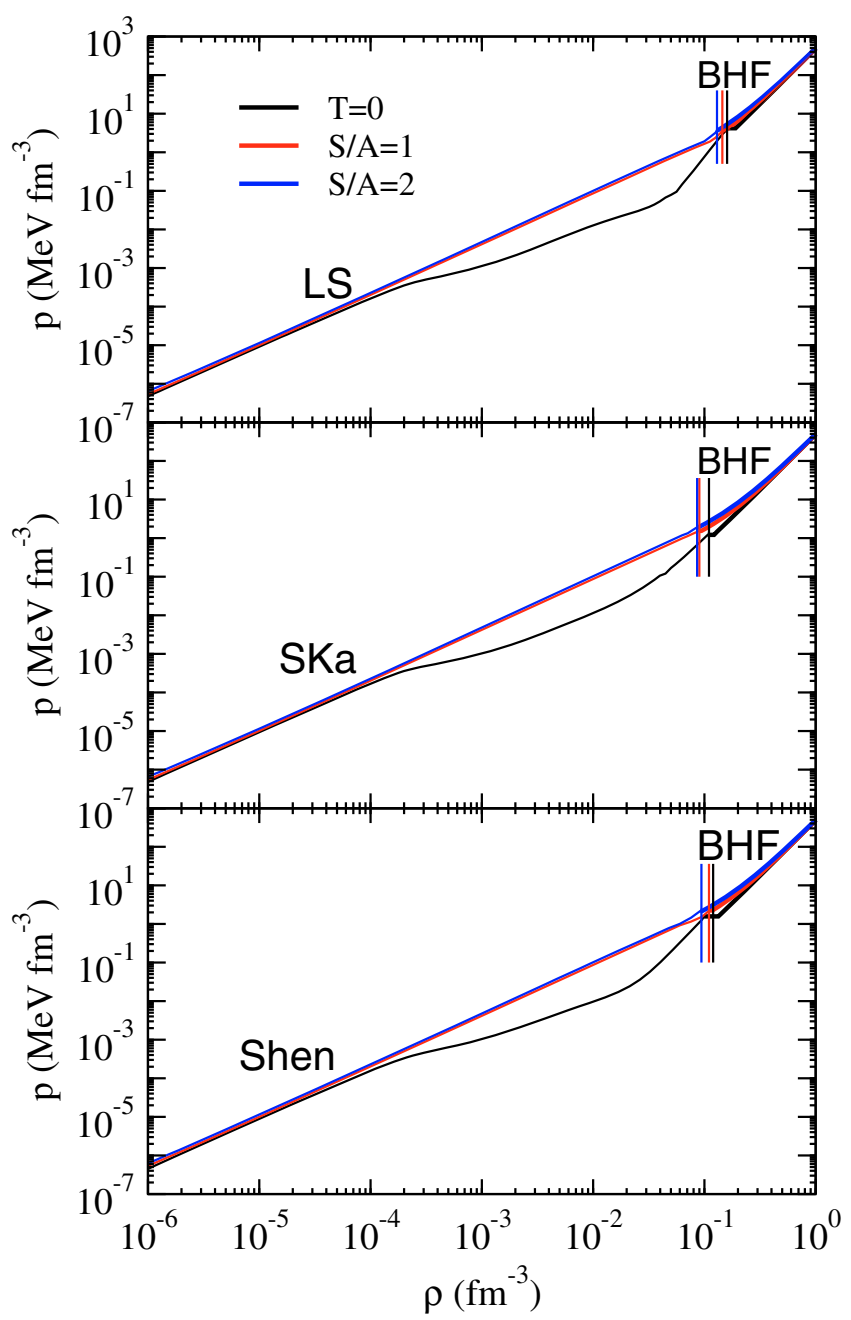

Fig. 7. The EOS for cold untrapped matter (black curves) and for neutrino-trapped matter with entropy $S / A=1$ (red curves) and $S / A=2$ (blue curves).

Table 2. Properties of (P)NS minimum and maximum mass configurations.

\begin{tabular}{lllllccc}
\hline \hline & \multicolumn{3}{c}{ Mminimum mass } & \multicolumn{3}{c}{ Maximum mass } \\
& & $M / M_{\odot}$ & $R(\mathrm{~km})$ & $\rho_{\mathrm{c}} / \rho_{0}$ & $M / M_{\odot}$ & $R(\mathrm{~km})$ & $\rho_{\mathrm{c}} / \rho_{0}$ \\
\hline untrapped & LS & & & & 2.03 & 9.86 & 10.55 \\
$T=0$ & SKa & & & & 2.03 & 9.86 & 10.42 \\
& Shen & & & & 2.03 & 9.93 & 10.42 \\
\hline trapped & LS & 0.58 & 40 & 1.02 & 1.95 & 10.2 & 11.34 \\
$S / A=1$ & SKa & 0.60 & 38 & 1.08 & 1.95 & 10.2 & 11.20 \\
& Shen & 0.58 & 44 & 1.02 & 1.95 & 10.3 & 11.20 \\
\hline trapped & LS & 0.70 & 44 & 0.90 & 1.95 & 10.7 & 10.85 \\
$S / A=2$ & SKa & 0.77 & 42 & 0.90 & 1.95 & 10.8 & 10.70 \\
& Shen & 0.75 & 52 & 0.77 & 1.95 & 10.8 & 10.80 \\
\hline
\end{tabular}

\section{Conclusions}

We have presented a microscopic calculation of the EOS of hot nuclear matter in the BHF approach and provided convenient parametrizations of the free energy as a function of baryon density, proton fraction, and temperature. All other thermodynamic quantities of interest can be derived from these. This EOS was joined to different standard EOSs describing the low-density, inhomogeneous domain of hot nuclear matter, and we roughly modeled the structure of PNSs, using strongly idealized temperature and neutrino-trapping profiles.

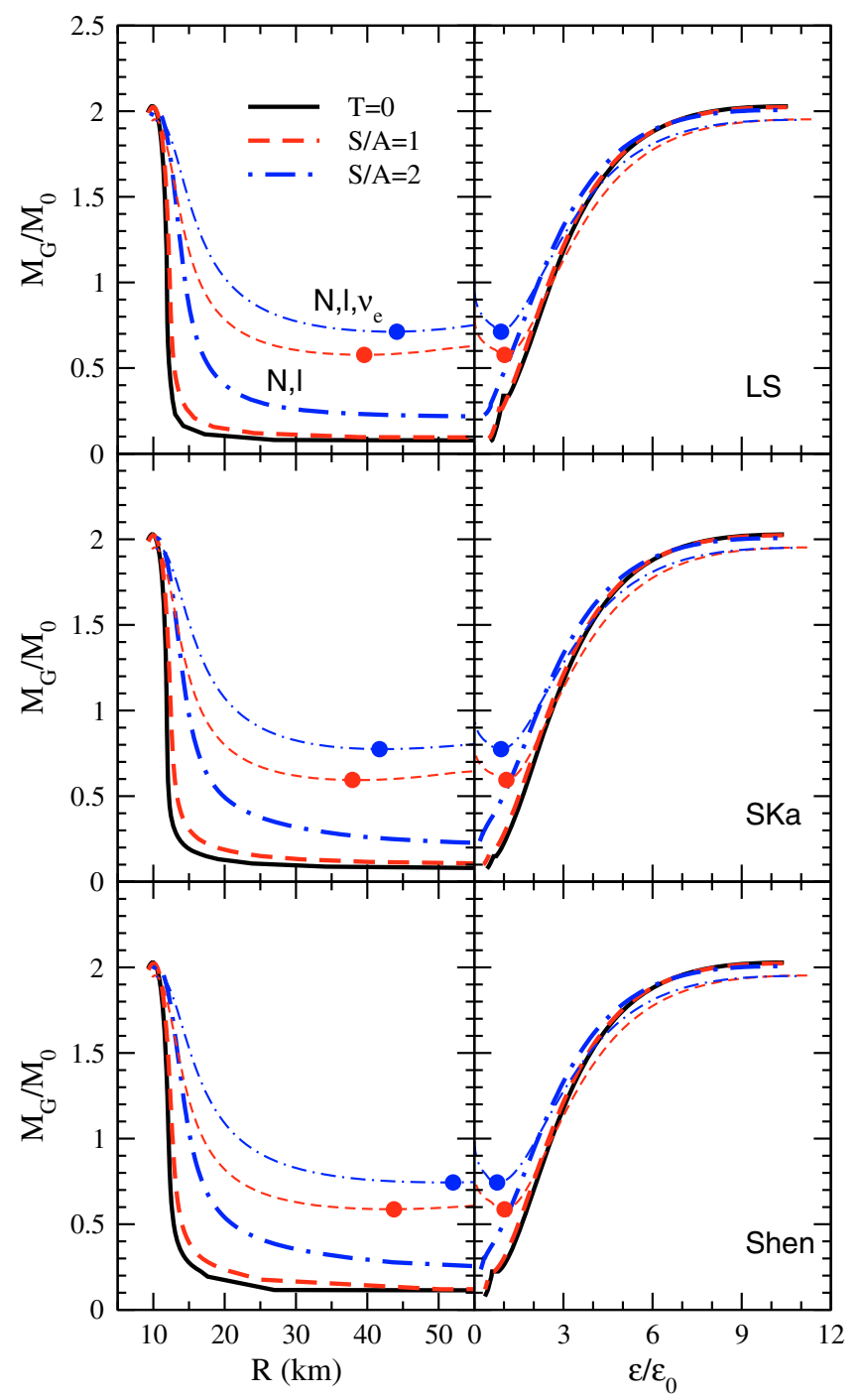

Fig. 8. The gravitational mass as a function of the radius (left panels), and the central energy density (right panels), obtained with the LS (top panels), SKa (central panels), and Shen (bottom panels) EOSs.

Altogether we find only small variations in our results for the minimum mass configurations when different low-density EOSs are employed, while maximum masses are practically unaffected. Both neutrino trapping and (to a minor degree) finite temperature decrease the asymmetry of nuclear matter, and thus soften the EOS and decrease the maximum mass.

However, to provide more quantitative results, it will be necessary to perform consistent dynamical simulations of the PNS evolution, using self-consistent temperature and neutrinotrapping profiles, along with the temperature dependent EOS. This is a difficult task for the future.

Acknowledgements. We thank M. Baldo, V. Ferrari, and L. Gualtieri for useful discussions. We acknowledge the support of COMPSTAR, a research and training program of the European Science Foundation.

\section{References}

Baldo, M. 1999, Nuclear Methods and the Nuclear Equation of State (Singapore: World Scientific)

Baldo, M., \& Ferreira, L. S. 1999, Phys. Rev. C, 59, 682

Baldo, M., Bombaci, I., \& Burgio, G. F. 1997, A\&A, 328, 274

Baldo, M., Burgio, G. F., \& Schulze, H.-J. 1998, Phys. Rev. C, 58, 3688

Baldo, M., Burgio, G. F., \& Schulze, H.-J. 2000a, Phys. Rev. C, 61, 055801 
Baldo, M., Song, H. Q., Giansiracusa, G., \& Lombardo, U. 2000b, Phys. Lett. B, 473,1

Baldo, M., Fiasconaro, A., Song, H. Q., Giansiracusa, G., \& Lombardo, U. 2001, Phys. Rev. C, 65, 017303

Bloch, C., \& De Dominicis, C. 1958, Nucl. Phys., 7, 459

Bloch, C., \& De Dominicis, C. 1959a, Nucl. Phys., 10, 181

Bloch, C., \& De Dominicis, C. 1959b, Nucl. Phys., 10, 509

Bombaci, I., \& Lombardo, U. 1991, Phys. Rev. C, 44, 1892

Burgio, G. F., \& Schulze, H.-J. 2009, Phys. Atomic Nuclei, 72, 1197

Burrows, A., \& Lattimer, J. M. 1986, ApJ, 307, 178

Carlson, J., Pandharipande, V. R., \& Wiringa, R. B. 1983, Nucl. Phys. A, 401, 59

Fischer, T., Whitehouse, S. C., Mezzacappa, A., Thielemann, F.-K., \& Liebendörfer, M. 2009, A\&A, 499, 1

Gondek, D., Haensel, P., \& Zdunik, J. L. 1997, A\&A, 325, 217

Goussard, J. O., Haensel, P., \& Zdunik, J. L. 1997, A\&A, 321, 822

Lattimer, J. M., \& Swesty, F. D. 1997, Nucl. Phys. A, 535, 331, http://www. astro. sunysb. edu/lattimer/EOS/main.html

Li, Z. H., Lombardo, U., Schulze, H.-J., et al. 2006, Phys. Rev. C, 74, 047304

Li, Z. H., Lombardo, U., Schulze, H.-J., \& Zuo, W. 2008a, Phys. Rev. C, 77, 034316

Li, B. A., Chen, L. W., \& Ko, C. M. 2008b, Phys. Rep., 464, 113

Liebendörfer, M., Rampp, M., Janka, H.-Th., \& Mezzacappa, A. 2005, ApJ, 620, 840
Nicotra, O. E., Baldo, M., Burgio, G. F., \& Schulze, H.-J. 2006, A\&A, 451, 213 Pons, J. A., Reddy, S., Prakash, M., Lattimer, J. M., \& Miralles, J. A. 1999, ApJ, 513,780

Prakash, M., Bombaci, I., Prakash, M., et al. 1997, Phys. Rep., 280, 1

Schiavilla, R., Pandharipande, V. R., \& Wiringa, R. B. 1986, Nucl. Phys. A, 449, 219

Schulze, H.-J., Lejeune, A., Cugnon, J., Baldo, M., \& Lombardo, U. 1995, Phys. Lett. B, 355, 21

Schulze, H.-J., Lejeune, A., Cugnon, J., Baldo, M., \& Lombardo, U. 1998, Phys. Rev. C, 57, 704

Shapiro, S. L., \& Teukolsky, S. A. 1983, Black Holes, White Dwarfs, and Neutron Stars (New York: John Wiley \& Sons)

Shen, H., Toki, H., Oyamatsu, K., \& Sumiyoshi, K. 1998a, Nucl. Phys. A, 637, 435

Shen, H., Toki, H., Oyamatsu, K., \& Sumiyoshi, K. 1998b, Prog. Theor. Phys., 100, 1013, http://user. numazu-ct.ac.jp/ sumi/eos/index.html

Song, H. Q., Baldo, M., Giansiracusa, G., \& Lombardo, U. 1998, Phys. Rev. Lett., 81, 1584

Strobel, K., Schaab, C., \& Weigel, M. K. 1999, A\&A, 350, 497

Villain, L., Pons, J. A., Cerdá-Durán, P., \& Gourgoulhon, E. 2004, A\&A, 418, 283

Wiringa, R. B., Stoks, V. G. J., \& Schiavilla, R. 1995, Phys. Rev. C, 51, 38

Zhou, X. R., Burgio, G. F., Lombardo, U., Schulze, H.-J., \& Zuo, W. 2004, Phys. Rev. C, 69, 018801 